\title{
Empowering One-vs-One Decomposition with Ensemble Learning for Multi-Class Imbalanced Data
}

\author{
Zhongliang Zhang ${ }^{\mathrm{a}}$, Bartosz Krawczyk ${ }^{\mathrm{b}, *}$, Salvador Garcìa ${ }^{\mathrm{c}}$, Alejandro \\ Rosales-Pérez ${ }^{\mathrm{d}}$, Francisco Herrera ${ }^{\mathrm{c}, \mathrm{e}}$ \\ ${ }^{a}$ School of Information Science and Engineering, Key Laboratory of Integrated \\ Automation of Process Industry, Northeastern University, Shenyang 110819, China. \\ ${ }^{b}$ Department of Systems and Computer Networks, Wroctaw University of Technology, \\ Wyb. Wyspiańskiego 27, 50-370 Wroctaw, Poland. \\ ${ }^{c}$ Department of Computer Science and Artificial Intelligence, University of Granada, \\ P.O. Box 18071, Granada, Spain. \\ ${ }^{d}$ Instituto Nacional de Astrofísica, Óptica y Electrónica, Computer Science Department, \\ Luis E. Erro No. 1, Santa María Tonantzintla, Puebla, 72840, Mexico. \\ ${ }^{e}$ Faculty of Computing and Information Technology, King Abdulaziz University, 21589, \\ Jeddah, Saudi Arabia.
}

\begin{abstract}
Multi-class imbalance classification problems occur in many real-world applications, which suffer from the quite different distribution of classes. Decomposition strategies are well-known techniques to address the classification problems involving multiple classes. Among them binary approaches using one-vs-one and one-vs-all has gained a significant attention from the research community. They allow to divide multi-class problems into several easier-to-solve two-class sub-problems. In this study we develop an exhaustive empirical analysis to explore the possibility of empowering the one-vsone scheme for multi-class imbalance classification problems with applying binary ensemble learning approaches. We examine several state-of-the-art ensemble learning methods proposed for addressing the imbalance problems to solve the pairwise tasks derived from the multi-class data set. Then the aggregation strategy is employed to combine the binary ensemble outputs to
\end{abstract}

\footnotetext{
*Corresponding author

Email addresses: zzl19860210@126.com (Zhongliang Zhang), bartosz.krawczyk@pwr.edu.pl (Bartosz Krawczyk), salvagl@decsai.ugr.es (Salvador Garcìa), arosales@inaoep.mx (Alejandro Rosales-Pérez), herrera@decsai.ugr.es (Francisco Herrera)
}

Preprint submitted to Knowledge-Based Systems

April 1, 2016

(C) 2016. This manuscript version is made available under the Elsevier user license http://www.elsevier.com/open-access/userlicense/1.0/ 
reconstruct the original multi-class task. We present a detailed experimental study of the proposed approach, supported by the statistical analysis. The results indicate the high effectiveness of ensemble learning with one-vs-one scheme in dealing with the multi-class imbalance classification problems.

Keywords: Multi-class classification, Imbalanced data, Ensemble learning, Binary decomposition, Classifier combination

\section{Introduction}

In machine learning and data mining, while one or more classes are underrepresented in the data set, it is called as class imbalance classification. Many real-world classification tasks suffer from the class imbalance problem, which is considered as one of the important challenges for the data mining community [18]. The main difficulty of these problems is that the skewed distribution makes conventional classification algorithms less effective, since standard learning algorithms consider a balanced training data set, which result in making it harder to predict minority class examples [50].

In recent years, many efforts have been focused on the binary class imbalance problems $[31,41]$, which only contain two classes. However, multi-class imbalance classification, is widely applied in many areas, such as text categorization [47], human activity recognition [1] and medical diagnosis [35]. Unfortunately, it may be invalid to directly apply the solutions proposed for the two-class problems to the multi-class imbalance problems, and some algorithms cannot be used to solve the multi-class imbalance problems directly [18].

Fortunately, in the research community, decomposition strategies turn up to deal with multi-class classification problem. In this solution framework, the multi-class classification problems are transformed into binary class sub-problems, which are much easier to discriminate $[61,53]$. Such wellknown approaches are the one versus one (OVO) [33, 25] and one versus all (OVA) [7]. As OVA introduces an artificial class imbalance (e.g., for 10 class problem with roughly equally represented classes, the binary sub-problem will have an imbalance ratio 1:9), it is not advisable to use it for handling problems with initially skewed distributions [46].

In this paper, we focus on multi-class imbalance classification problems and develop a complete empirical study to explore the effectiveness of ensemble learning methods [62] in the multi-class imbalanced datasets with 
OVO scheme, where binary-class classifiers are trained from the subset containing each pair of classes by ensemble learning approaches based on data preprocessing [23]. Our initial works in this domain showed that empowering binary decomposition with pairwise ensemble learning can significantly improve mining imbalanced multi-class problems [34].

Regarding ensemble learning methods, six state-of-the-art approaches are selected to carry out the experiment: UnderBagging [3], SMOTEBagging [56] [15], RUSBoost [49], SMOTEBoost [10], SMOTE+AdaBoost [40], EasyEnsemble [40]. Additionally, to show the efficiency of ensemble learning with OVO scheme for addressing the multi-class imbalance problems, the original data preprocessing strategies, including random under-sampling (RUS) [4], random over-sampling (ROS) [4] and synthetic minority oversampling technique (SMOTE) [8], are also implemented in the OVO scheme for our comparative analysis.

Finally, we carry out a thorough experimental study that supports the effectiveness of our methodology. Concretely, 20 multi-class imbalanced data sets are selected from the UCI repository in our experiment. The average accuracy rate [20] is used as the performance measures in this study. In order to analyze the results obtained from the different solutions, statistical analysis suggested in [28] is given to support the significance of the results.

The main contributions of this paper with respect to previous studies are as following:

- We propose to enhance the OVO scheme for multi-class imbalanced data by using ensemble techniques for each sub-problem.

- We show, how to extend the area of applicability of binary imbalanced ensemble classifiers to handling far more challenging multi-class imbalanced scenarios.

- We develop a complete experimental study of comparison of the stateof-the-art ensemble learning techniques with conventional resampling methods with OVO strategy and state-of-the-art solutions for multiclass imbalance problems.

- In order to obtain the impacts of the base classifier used in our scenario, we choose three different algorithms, including Classification and Regression tree (CART) [6], Back Propagation Neural Network (BPNN) [17] and Support Vector Machine (SVM) [54]. 
The rest of this paper is organized as follows. The background of this study is introduced in Section 2, including multi-class imbalance classification problems and decomposition strategies. Next, in Section 3 we present the framework of our methodology of ensemble learning with OVO scheme for dealing with multi-class imbalance classification problems. In section 4 , the experimental framework is given, including the data sets, the base classifiers and the relative parameters setting, the performance measures and the statistical tests. The complete empirical study is presented in Section 5. Lessons learned from the paper are given in Section 6, while conclusions and potential directions for future works are to be found in the final Section.

\section{Background}

In this section, we first introduce the problem of multi-class imbalance classification. Then, we present the solutions for addressing the imbalance problems. Finally, we describe the decomposition strategy for dealing with multi-class classification problems.

\subsection{Multi-class Imbalanced Data Analysis}

Multi-class imbalanced data sets, where there are much more instances of some classes (referred to as the majority classes) than others (referred to as the minority classes), is one of the most challenging problems with data quality that always reduces classification performance in machine learning and data mining [57]. The minority classes are usually the most important concepts to recognize, since they represent the rare cases [59]. Additionally, it is expensive or hard to select these examples [58].

However, standard classification algorithms are designed with the premise of a balanced training set [42]. With such a precondition, it is much more difficult for the classical classification algorithms to deal with class imbalance problems, especially for identifying the minority class instance [9]. Additionally, most of the methods however are specific to address the binary class imbalance problems. Obviously, multi-class imbalance problems are far more complex, since these issues are involved with large number of classes and the relationships among the classes are complicated. Furthermore, it is hard to distinguish between minority classes and noise examples and the minority classes can be ignored by the classifier as the noise examples. 


\subsection{Solutions for Imbalanced Classification Problems}

To overcome the dilemma of skewed class distribution, a large amount of techniques have been developed to deal with such problem. These proposals can be roughly categorized into four groups:

- Data level: the origin of the problem is the class distribution in the data sets, therefore, it is natural to consider of rebalancing by sampling the data space to reduce the impact of class imbalance, known as an external approach. One of the advantages of such solution is independent from the classifier used, so they are also considered as pre-sampling method $[27,51]$.

- Algorithm level: these solutions try to adopt appropriate decision threshold to reinforce the learning towards the minority class instances. The proposed algorithms that take the class imbalance into consideration belong to such techniques. They are defined as internal approaches in some papers $[52,11]$, since the effect depends on the problems and the classifier [13]. One of the most well-known solutions is the direct modification of the learning procedure for a selected algorithm [45].

- Cost-sensitive level: these approaches consider higher costs for misclassifying the minority classes with respect to the majority classes, that is, misclassification of minority class is much more expensive [44]. The learning process turns to minimize the cost errors instead of maximization of accuracy rate [63].

- Ensemble level: these solutions combine the efficient ensemble learning solutions [62] with one of the three previously mentioned strategies in order to create a balanced training sets for base classifier and at the same time introduce diversity into the pool of base learners. Special attention should be paid to recent combination of intelligent and directed data-level approaches with Bagging solution [5] or randomized oversampling [15], hybrid combination of algorithm-level methods [55] and cost-sensitive pruning for decision tree ensembles [36].

Due to the advantage of the data level solutions (as pointed out by a recent tutorial on data preprocessing [29]) we focus on such methods in this study. 
RUS [51] is the basic under-sampling, which randomly removes the majority class instances to balance the class distribution. This approach is efficient for dealing with class imbalance problems, since most of the majority class instances are redundant. Additionally, RUS makes the training process become much faster, since the training set contains less instances than original data set. However, some potential useful information contained in the majority class instance may be neglected, since RUS randomly generates the subset without considering the relationship among the instances.

ROS [27] is another basic resampling approach, which randomly duplicates the minority class instances to make the training set balanced. With such consideration, the size of minority class examples tends to match the size of the other classes. However, it might lead to two important defects. One of the problems is overfitting, since ROS typically replicates examples of the minority class so that the interface between minority class and majority class is too prone to the former. Another problem is that ROS increases the training time. Suppose there is an imbalance data set with 20000 majority class examples and 100 minority class examples, to generate an equal size of minority class and majority class, the ROS will create a training data set with 40000 examples. Obviously, it must take much more time if ROS is used as resampling technique.

SMOTE is an intelligent oversampling approach proposed by Chawla et al. [8]. Unlike ROS duplicates the minority examples, SMOTE produces synthetic minority class examples by $k$ nearest neighbors, augmented with randomized interpolation. However, the noise might incorporate in the synthetic minority class examples.

In the above reviewed methods, sampling process is independently carried out before the training process. In these approaches, the training process is unchanged and the classifiers are trained by the balanced data sets produced in the resampling process. To overcome the drawbacks of classical resampling approaches mentioned above, some ensemble learning methods [37] are proposed to devote to combine the resampling process and training process. These techniques developed for dealing with class imbalance problems follow the architecture of bagging or boosting.

In the framework of bagging, the base classifier is trained by using the resampling examples. That is, the diversity is obtained through resampling minority class instances, since bootstrapped instances of the original data set are randomly duplicated. Many proposals are introduced in the research community, such as OverBagging, UnderBagging, and UnderOverBagging [3]. 
Regarding to the scenario of boosting, the resampling techniques are embed into bootstrap. In such a manner, the resampling methods are introduced in each iteration to train a classifier toward the minority class. In this family, SMOTEBoost [10] and RUSBooost [49] are the representative proposals. Besides these techniques, some algorithms combine both bagging and boosting to obtain an ensemble of ensembles [40].

However, when we refer to multi-class imbalance classification problems, the conventional solutions may not be feasible or perform a lower achievement. There are still only few approaches for handling this task. StaticSMOTE [19] applied resampling procedure in $M$ steps, where $M$ is the number of classes. In each iteration, the resampling procedure selects the minimum size class, and duplicates the number of instances of the class in the original data-set. An ensemble learning algorithm for multi-majority and multi-minority cases was proposed in [57]. Authors combine AdaBoost with negative correlation learning, where starting weights of examples are calculated in inverse proportion to the number of objects in this class. A combination of binary decomposition and pre-processing methods was proposed as an efficient solution when the number of classes is high [18].

More recent studies on this problem propose to combine pairwise modified SVMs with boundary shift asymmetric regularization costs using one-vs-all technique [14]. Additionally, two hybrid ensemble techniques with embedded feature selection were proposed to deal with the problem of skewed distributions among multiple classes [30,38]. The most recent work study in this domain reports the high importance of considering the individual types of minority classes examples and their learning difficulty when performing oversampling for multi-class imbalanced data and proposes a data-driven universal strategy that can be embedded in any data-level multi-class solution [48].

As one can see, there are many efficient ensemble techniques dedicated to binary problems. However, it is not straightforward to extend them for scenarios with higher number of classes. Therefore, the ensemble learning methods are considered in this study to learn the pattern from the data set derived from paired classes, and then the binary ensembles are combined by aggregation strategy to be a final multi-class classifier.

\subsection{One-vs-One Scheme for Multi-Class Classification Problems}

In the OVO decomposition scheme, a $m$-class problem is divided into $m(m-1) / 2$ binary subproblems. Each problem is faced by independent base classifiers, which are responsible for distinguishing the instances from 
the different pairs of classes. With such appropriate consideration, the much more complex multi-class problem is translated into the simpler binary class subproblems, which is expected to receive better results or address multi-class problems with binary classification techniques. An example of binarization technique for decomposing the multi-class problem is shown in Figure 1.

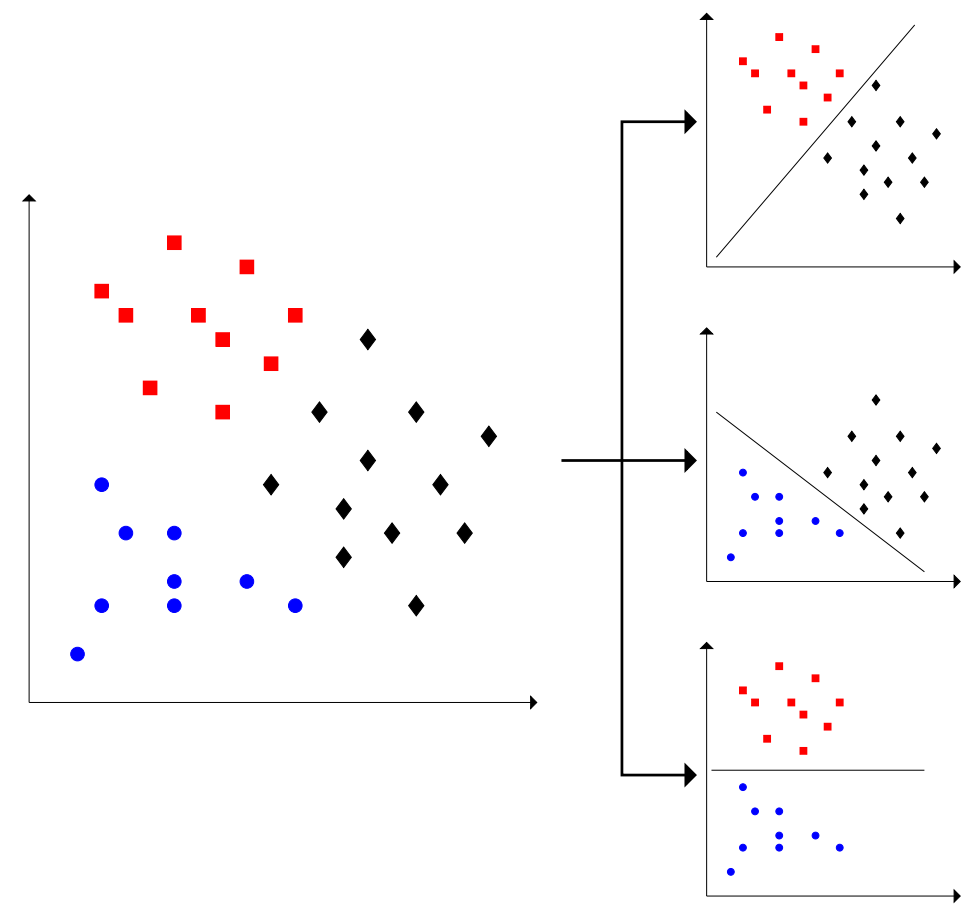

Figure 1: An example of one-versus-one (OVO) decomposition of a three-class problem into three two-class problems.

In order to predict a new pattern, there are two phases required to complete the task. The first phase is to learn the classifiers that are trained by the original instances with pairwise classes, that is, the task of each classifier is to distinguish a pair of classes $\left\{C_{i}, C_{j}\right\}$. In the integration phase, a confidence degree $r_{i j} \in[0,1]$ in favor of $C_{i}$ is given by a classifier to discriminate the class $i$ from class $j$. The confidence in favor of $j$-th class is computed as $r_{j i}=1-r_{i j}$, if the classifier considers the output class as the class with the largest confidence value. To be clearer, all the confidence degree can be represented by a score matrix $\mathrm{R}$ : 


$$
R_{m, n}=\left(\begin{array}{cccc}
- & r_{1,2} & \cdots & r_{1, m} \\
r_{2,1} & - & \cdots & a_{m, n} \\
\vdots & \vdots & \ddots & \vdots \\
r_{m, 1} & r_{m, 2} & \cdots & -
\end{array}\right)
$$

There are a number of aggregations proposed in the literature [26, 39] to infer the final class. The voting strategy (VOTE) is the simplest but powerful aggregation, therefore, it is considered as the aggregation approach in this study. VOTE, which is also called binary voting and Max-Wins rule [21], considers a vote for the predicted class by the binary classifier. Votes received by each class are counted and the final class obtaining the largest number of votes is predicted as follows:

$$
\text { Class }=\arg \max _{i=1, \cdots, m} \sum_{1 \leq j \neq i \leq m} s_{i j},
$$

where

$$
s_{i j}=\left\{\begin{array}{ll}
1 & r_{i j}>r_{j i} \\
0 & \text { otherwise }
\end{array} .\right.
$$

\section{Combining OVO with Ensemble Learning for Multi-class Imbal- anced Data}

As mentioned above, on the one hand, ensemble learning proposed for imbalanced datasets may be not effective or even impossible for dealing with multi-class problems. On the other hand, decomposition strategy is effective direction to handle the multi-class classification problems. Therefore, it is interesting for us to consider their respective advantages in the scenario of multi-class imbalanced datasets. In this section, we firstly describe our methodology of employing the ensemble learning approaches in the OVO scheme. Then, we present the well-known ensemble learning methods employed in this study.

\subsection{Solving Multi-Class Imbalanced Problems with Binary Decomposition}

Multi-class imbalance classification problems are extremely complex tasks, which suffer from more than two classes and class imbalance distribution. According to the suggestion of decomposition strategy, which aims at solving 
the complex problem by decomposing it into series of much easier subproblems, we propose to explore the effectiveness of combination of ensemble learning and OVO scheme to address the multi-class imbalance classification problems.

Firstly, the multi-class imbalance classification problems are decomposed into a series of pairwise datasets. One should note that there are some pairwise classes after decomposition will have roughly equal size. For these cases, the subproblems are handled as normal binary classification problems by the normal ensemble learning algorithms. Then, the ensemble learning methods based on the resampling techniques devote to address the binary class imbalance datasets.

In this work we focus only on OVO approach as recent studies on decomposition techniques clearly proved its superiority over OVA methods [22, 24]. Additionally, studies on combination of resampling strategies with single classifiers for multi-class imbalanced data showed clearly that OVA displays inferior performance [18]. Finally, OVA introduces an artificial class imbalance which may further damage the learning process in scenarios with multiple skewed distributions.

An example of the proposed approach is shown in Figure 2.

There are two strategies employed in the ensemble learning process.

As shown in Figure 2 (left), one of the ensemble learning algorithms combines the single classifiers which are derived from the balanced datasets by reducing the size of the majority class. Concretely, the multi-class classification problem is split into several binary class subproblems. Then, the ensemble learning method combining under-sampling strategy is employed to learn a binary classifier for each subproblem. Finally, the aggregation strategy is considered to obtain the final output class.

As described in Figure 2 (right), oversampling approach is adopted to increase the number of the minority class to balance the class distribution. Firstly, the binary class subproblems are derived from the original multiclass classification problem. Secondly, ensemble learning method considering SMOTE to create the synthetic minority class to balance the distribution for the training process is used to achieve a binary classifier for each pairwise class data set. Finally, once each binary classifier derived from the ensemble learning, aggregation strategy will be employed to provide the final output from the score matrix.

Let us now discuss ensemble learning algorithms used in this study. 


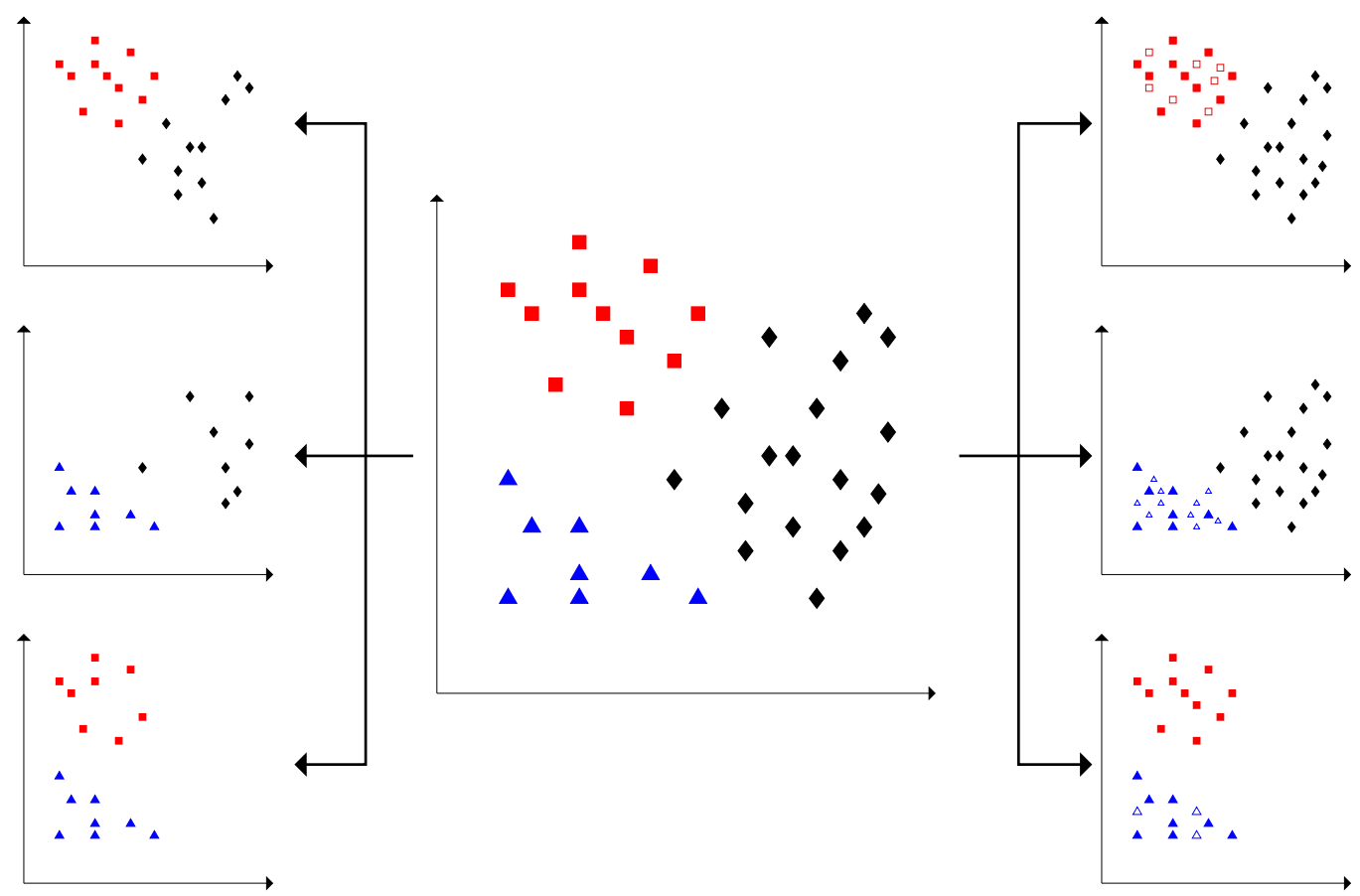

Figure 2: An example of pre-processing methods applied over a decomposed multi-class imbalanced dataset. (Left) Undersampling applied to each subproblem. (Right) Oversampling applied to each subproblem.

\subsection{Ensemble Learning Algorithms for Binary Imbalanced Data}

As described above, ensemble learning is applied in our study to classify the binary classes which are derived from the multiclass data sets. Therefore, several well-known ensemble learning algorithms proposed for imbalance problems were selected to train the binary classifiers. Specifically, the ensemble learning algorithms employed in this study are as follows:

- UnderBagging (UBA). RUS is applied to under-sampling the majority class in each bag of the ensemble. In this way, we can obtain the balanced data set for each classifier of the bag and the diversity for each bag is produced with different majority class instances and the same minority class instances. Additionally, the strategy of resampling with replacement is adopted in order to increase the diversity among the bags.

- SMOTEBagging (SBA). SMOTE algorithm is employed to insert ar- 
tificial minority class in each bag. This way aims at balancing the distribution of the data set by increasing the number of minority class instances. The diversity of each bag of the ensemble is obtained with mutative synthetic samples.

- RUSBoost (RBO). This strategy applies RUS of minority class to balance the distribution of the data set before ensemble learning. The AdaBoost is employed to construct a classifier from the balanced data set. One should note that this method handle the imbalance problem before the ensemble learning process.

- SMOTEBoost (SBO). SMOTE algorithm is employed to balance the distribution of the data set in each iteration of AdaBoost. Specifically, a training set is obtained by resampled from weighted samples. Then, synthetic samples are generated to increasing the number of minority class. Therefore, the distribution of the training set for each single classifier in AdaBoost is balanced.

- SMOTE+AdaBoost (SMB). Considering to this method, we apply SMOTE algorithm to generate synthetic majority class to balance the data set before AdaBoost. That is, the SMOTE is independent from AdaBoost, which is different from SBO employing SMOTE algorithm during Boosting progress.

- EasyEnsemble (Easy). Under-sampling is simple but effective for handling imbalance problem, since the training data set is much more balanced and the training process is much faster. However, many majority class examples are ignored, which leads to loss potentially useful information. In order to overcome the drawback, this method constructs classifier ensembles from all of the minority class and a subset of the majority class. Then, the final ensemble combines the outputs of classifiers which are built by using AdaBoost algorithm. Therefore, it can be considered as an ensemble of ensembles.

\section{Experimental Framework}

In this section, we present the set-up of the experimental framework used in the experiment of Section 5. The data sets chosen to test the algorithms are described in Section 4.1, while single and ensemble classifiers with their 
Table 1: Summary description of the data sets used in the experimental study.

\begin{tabular}{|c|c|c|c|c|c|c|c|c|}
\hline id & Dataset & $\# \mathrm{Ex}$. & \#Atts. & \#Num. & \#Nom. & $\# \mathrm{Cl}$. & \#Dc. & IR \\
\hline Aut & Automobile & 159 & 25 & 15 & 10 & 6 & $46 / 29 / 13 / 48 / 20 / 3$ & 16.00 \\
\hline Bal & Balance & 625 & 4 & 4 & 0 & 3 & $288 / 49 / 288$ & 5.88 \\
\hline Car & Car & 1728 & 6 & 0 & 6 & 4 & $384 / 69 / 1210 / 65$ & 18.62 \\
\hline Cle & Cleveland & 297 & 13 & 5 & 8 & 5 & $160 / 54 / 35 / 35 / 13$ & 12.31 \\
\hline Con & Contraceptive & 1473 & 9 & 6 & 3 & 3 & $629 / 333 / 511$ & 1.89 \\
\hline Der & Dermatology & 358 & 34 & 1 & 33 & 6 & $111 / 60 / 71 / 48 / 48 / 20$ & 5.55 \\
\hline Fla & Flare & 1066 & 11 & 0 & 11 & 6 & $147 / 211 / 239 / 95 / 43 / 331$ & 7.70 \\
\hline Gla & Glass & 214 & 9 & 9 & 0 & 6 & $70 / 76 / 17 / 29 / 13 / 9$ & 8.44 \\
\hline Hay & Hayes-roth & 160 & 4 & 4 & 0 & 3 & $65 / 64 / 31$ & 2.10 \\
\hline Led & Led7digit & 500 & 7 & 0 & 7 & 10 & $45 / 37 / 51 / 57 / 52 / 52 / 47 / 57 / 53 / 49$ & 1.54 \\
\hline Lym & Lymphography & 148 & 18 & 3 & 15 & 4 & $4 / 61 / 81 / 2$ & 40.50 \\
\hline New & New-thyroid & 215 & 5 & 5 & 0 & 3 & $150 / 35 / 30$ & 5.00 \\
\hline Pag & Page-blocks & 5472 & 10 & 10 & 0 & 5 & $4913 / 329 / 28 / 87 / 115$ & 175.46 \\
\hline Sat & Satimage & 6435 & 36 & 36 & 0 & 6 & $1533 / 703 / 1358 / 626 / 707 / 1508$ & 2.45 \\
\hline Shu & Shuttle & 2175 & 9 & 9 & 0 & 5 & $1706 / 2 / 6 / 338 / 123$ & 853.00 \\
\hline Spl & Splice & 3190 & 60 & 0 & 60 & 3 & $767 / 768 / 1655$ & 2.16 \\
\hline Thy & Thyroid & 720 & 21 & 6 & 15 & 3 & $17 / 37 / 666$ & 39.18 \\
\hline Win & Wine & 178 & 13 & 13 & 0 & 3 & $59 / 71 / 48$ & 1.48 \\
\hline Wqr & Wine-Quality-Red & 1599 & 11 & 11 & 0 & 6 & $10 / 53 / 681 / 638 / 199 / 18$ & 68.10 \\
\hline Zoo & Zoo & 101 & 16 & 0 & 16 & 7 & $41 / 20 / 5 / 13 / 4 / 8 / 10$ & 10.25 \\
\hline
\end{tabular}

parameters are described in Section 4.2. The measure to evaluate the performance of the approaches in this study are presented in Section 4.3. Final Section describes the statistical test applied to compare the results obtained from experiments.

\subsection{Data Sets}

In this study, twenty data sets from the UCI repository were selected to test the methodology. The properties of the data sets were showed in Table 1. For each data set, it includes the number of examples (\#Ex.), the number of attributes (\#Atts.), the number of numerical (\#Num) and nominal (\#Nom) attributes, the number of classes (\#Cl.), the distribution of class (\#Dc) and the imbalance rate (IR). For the missing values instances in the data sets (Cleveland and Dermatology), we removed them before doing the partitions.

The results of the average accuracy was obtained by means of 5 times 5 -fold stratified cross-validation (SCV) [43]. That is, each data set is split into 5 folds and each fold contained $20 \%$ of the instances of the data set. For each fold, the algorithm is trained with the instances contained in the remaining folds ( $80 \%$ of the instances of the data set) and then tested by the current fold. The reason why 5 -fold SCV is more appropriate than a 10 -fold $\mathrm{SCV}$ in such framework was explained in [42]. If we use smaller partitions, 
there would be some test sets without containing any instance from some of the classes.

\subsection{Classification Algorithms}

In this section, the base classification algorithms compared in the experiment are presented. Specially, CART, BPNN and SVM are selected for the study, and they are described as follows.

CART is a tree induction technique. CART is a binary recursive partitioning methodology to generate a tree, that is, a node in the tree always split the attribute into only two parts (nodes). In CART, the Gini index is used to measure the impurity of node. The attribute and the corresponding binary split on that maximizes the reduction in impurity are chosen as the splitting point. The process will continue until reach the stopping criterion, including there is no possible split point or the maximal tree is obtained. Due to the noise or outlier in the training data set, some branches reflect abnormal information, which may lead to overfit the data. Pruning is an effective way to avoid overfitting. In CART, the cost complexity pruning algorithm which is a post-pruning method is used to prune the tree. In the approach, the cost complexity of a tree is measured by a function of error rate, which is the percentage of instances misclassified by the tree.

BPNN is a typical feed forward neural network, which has input layer, hidden layer and output layer. Back propagation learns by iteratively processing a set of training samples, comparing the networks prediction for each sample with the actual known target value. For each training sample, the weights are modified so as to minimize the mean-square error between the networks prediction and the actual target value. These modifications are made in the backwards direction through each hidden layer down to the first hidden layer.

SVM is an effective machine learning method, which is based on VapnikChervonenkis structural risk minimization instead of the empirical risk. SVM maps the original input feature space into a high dimensional feature space in order to construct an optimal separating hyperplane with maximal margin. The choice of a proper kernel has a strong effect on the final quality of the obtained model [12].

These classifiers are used as base learners for six ensemble methods described in Section 3.2.

Additionally, to offer a fair comparison with other ensemble techniques dedicated to multi-class imbalanced learning we have selected two state-of- 
the-art methods: Near-Bayesian Support Vector Machines (NBSVM) [14] and multi-class imbalanced Boosting [57].

The first approach modifies the SVM classifier by using samples from two classes in order to achieve boundary shift and combines it with the asymmetric regularization costs. Additionally, authors propose a modification of the popular SMO algorithm to train NBSVM. To handle multi-class scenarios a binary aggregation scenario with OVA technique is being used.

AdaBoost.NC combines the multi-class AdaBoost algorithm with negative correlation learning, where starting weights of examples are calculated in inverse proportion to the number of objects in this class. This way it is possible to capture varying relations between classes.

Detailed parameters of used methods are given in Table 2.

\subsection{Performance Measures}

There is a large amount of measures for the performance of algorithms in the imbalance classification problems, for example, precision, sensitivity, G-mean [3], F-measure [2], or AUC [16]. However, all of them are designed especially for the binary class problems. Standard metrics such as classification rate (accuracy rate) is an unreasonable measure in the multi-class imbalance classification problem, as it does not differentiate the classification rates from different classes. For example, in the data set of thyroid, a classifier can achieve a high accuracy rate of $92.5 \%$, if it recognizes all the instances as class 3 . There are some proposals for measures displaying a balanced performance on multiple classes, like multi-class AUC [32]. For this study we have decided to use the average accuracy metric.

The average accuracy gives the same weight to each class. It achieves the accuracy rate of each class independently, and then the final result is obtained by the average value. The average accuracy is computed as follows:

$$
\text { AveAcc }=\frac{1}{m} \sum_{i=1}^{m} \operatorname{TRP}_{i},
$$

where $m$ is the number of classes and $\mathrm{TRP}_{i}$ stands for the True Positive Rate of the $i$-th class.

\subsection{Statistical Analysis}

Statistical tests are important for analyzing the experimental results to extract the findings. In this paper the hypothesis testing techniques, which 
Table 2: Parameters setting for single and ensemble classifiers used in the study.

\begin{tabular}{|c|c|}
\hline Methods & Parameters \\
\hline \multicolumn{2}{|r|}{ Single classifiers } \\
\hline CART & $\begin{array}{l}\text { Prune }=\text { true } \\
\text { Method = classification } \\
\text { Impure nodes must have } 10 \text { or more examples to be split } \\
\text { The number of nodes in hidden layer }=10\end{array}$ \\
\hline $\mathrm{BPNN}$ & $\begin{array}{l}\text { Transfer function of hidden layer }=\text { logsig } \\
\text { Transfer function of output layer }=\text { logsig } \\
\text { Train epochs }=100\end{array}$ \\
\hline SVM & $\begin{array}{l}\mathbf{C}=1.0 \\
\text { Tolerance parameter }=0.001 \\
\gamma=0.0046 \\
\text { Kernel type }=\text { Gaussian radial basis function } \\
\text { Optimization method }=\text { SMO }\end{array}$ \\
\hline \multicolumn{2}{|r|}{ Ensemble classifiers } \\
\hline UBA & $\begin{array}{l}\text { The number of bags }=40 \\
\text { The number of resampling majority class }=\text { the number of minority class }\end{array}$ \\
\hline SBA & $\begin{array}{l}\text { The number of bags }=40 \\
\text { The number of nearest neighbors in } \mathrm{SMOTE}=5\end{array}$ \\
\hline $\mathrm{RBO}$ & $\begin{array}{l}\text { The number of iterations in AdaBoost }=40 \\
\text { The number of resampling majority class }=\text { the number of minority class }\end{array}$ \\
\hline $\mathrm{SBO}$ & $\begin{array}{l}\text { The number of iterations in AdaBoost }=40 \\
\text { The number of nearest neighbors in SMOTE }=5\end{array}$ \\
\hline SMB & $\begin{array}{l}\text { The number of iterations in AdaBoost }=40 \\
\text { The number of nearest neighbors in SMOTE }=5\end{array}$ \\
\hline Easy & $\begin{array}{l}\text { The number of subsets is } 4 \\
\text { The number of iteration in each AdaBoost ensemble is } 10\end{array}$ \\
\hline \multicolumn{2}{|r|}{ Algorithms for multi-class imbalance } \\
\hline AdaBoost.NC & $\begin{array}{l}\mathbf{C}=2.0 \\
\sigma \in[1,2,3,4,5,10,20,30,40,50] \text { - best selected for each dataset } \\
\text { Optimization method }=\text { modified SMO } \\
\text { classifier combination }=\text { OVA } \\
\text { The number of iterations }=51 \\
\alpha=2 \\
\text { base classifier }=\mathrm{C} 4.5\end{array}$ \\
\hline
\end{tabular}


are recommended in [28], are used to provide statistical support for the analysis of the results obtained by the experiment. Specially, for the pairwise comparisons, Wilcoxon signed-rank test [60] is adopted as a non-parametric statistical procedure to perform pairwise comparisons between ensemble learning and resampling approaches in OVO scheme and comparisons of the representation ensemble learning with OVO scheme and the state-of-the-art methods. Then, for the multiple comparisons, average aligned-rankings [28] of each method are adopted to compare the behavior of each method with respect to the others. Moreover, Friedman aligned-ranks test is used to check whether the best method (the control one) is significant better than others.

\section{Experimental Study}

In this section we develop a thorough empirical analysis in addressing the multi-class imbalance classification problems. We want to verify the effectiveness of the combination of ensemble learning and OVO scheme for the multi-class imbalance classification compared with classical approaches. Therefore, we develop the pairwise comparative study on analysis of ensemble learning approaches versus resampling techniques in the OVO scheme with different base classifiers, including CART, BPNN and SVM. Additionally, to show that using ensembles as base classifiers in OVO can efficiently empower learning from multi-class imbalanced data we present comparison with two state-of-the-art algorithms dedicated to this problem.

\subsection{Evaluating Ensemble Approaches with CART as Base Classifier}

The study for the CART decision tree is shown in Table 3, where we present all the results of average accuracy.

According to the best result stressed through bold-face, we can clearly find that the methods considering ensemble learning in the OVO scheme always receive better results in each data set. Observing the average performance, SBO obtains the best performance, followed by SMB and SBA.

The statistical study based on Wilcoxon test for CART is developed in Table 4.

Results of Wilcoxon test show that for CART classifier ensemble systems can significantly outperform resampling-based strategies with single classifier. However, there is no single ensemble strategy that is statistically significantly better than all of three resampling approaches tested at the same time. For example SBO method achieves excellent $p$-values in comparison 
Table 3: Average accuracy [\%] results for resampling and ensemble learning methods with CART as the base classifier. The best result is highlighted in bold.

\begin{tabular}{|c|c|c|c|c|c|c|c|c|c|}
\hline \multirow{2}{*}{ Data sets } & \multicolumn{3}{|c|}{ Resampling techniques } & \multicolumn{6}{|c|}{ Ensemble learning } \\
\hline & RUS & ROS & SMOTE & UBA & SBA & $\mathrm{RBO}$ & $\mathrm{SBO}$ & $\mathrm{SMB}$ & Easy \\
\hline Aut & 10.00 & 83.93 & 78.43 & 10.00 & 79.97 & 67.38 & 81.60 & 69.85 & 51.63 \\
\hline Bal & 54.49 & 58.24 & 56.43 & 64.90 & 56.51 & 71.40 & 58.35 & 66.27 & 73.13 \\
\hline Car & 93.87 & 94.64 & 91.93 & 95.72 & 93.87 & 96.53 & 97.34 & 97.56 & 97.57 \\
\hline Cle & 31.92 & 30.19 & 28.82 & 31.92 & 29.22 & 32.98 & 31.65 & 33.18 & 31.89 \\
\hline Con & 48.54 & 48.11 & 47.96 & 51.97 & 47.96 & 48.31 & 47.85 & 48.96 & 50.79 \\
\hline Der & 94.36 & 95.15 & 95.37 & 95.45 & 95.37 & 94.49 & 94.94 & 94.65 & 94.41 \\
\hline Fla & 63.28 & 62.52 & 61.20 & 63.46 & 61.11 & 62.65 & 60.58 & 62.60 & 63.03 \\
\hline Gla & 64.08 & 66.66 & 67.90 & 71.38 & 69.39 & 69.68 & 72.17 & 72.59 & 71.78 \\
\hline Hay & 86.07 & 85.86 & 85.76 & 85.86 & 85.76 & 85.54 & 85.15 & 85.24 & 86.15 \\
\hline Led & 69.77 & 69.95 & 70.13 & 69.69 & 70.13 & 70.20 & 70.48 & 70.78 & 70.83 \\
\hline Lym & 11.00 & 79.05 & 69.98 & 11.00 & 69.98 & 36.10 & 76.10 & 62.51 & 19.83 \\
\hline New & 92.80 & 89.70 & 90.37 & 93.48 & 91.75 & 93.24 & 91.72 & 93.69 & 94.43 \\
\hline Pag & 91.98 & 81.19 & 84.77 & 94.24 & 84.31 & 94.09 & 85.33 & 93.63 & 94.68 \\
\hline Sat & 83.82 & 84.25 & 84.23 & 87.97 & 86.36 & 90.02 & 89.41 & 90.20 & 89.83 \\
\hline Shu & 78.23 & 85.85 & 85.11 & 79.69 & 85.10 & 37.39 & 85.94 & 41.89 & 25.98 \\
\hline Spl & 92.74 & 92.68 & 92.89 & 94.50 & 93.33 & 95.51 & 95.65 & 95.64 & 95.64 \\
\hline Thy & 96.24 & 93.77 & 93.77 & 98.14 & 93.77 & 96.42 & 92.95 & 98.61 & 98.16 \\
\hline Win & 91.77 & 92.60 & 92.29 & 94.18 & 92.29 & 97.32 & 91.34 & 96.60 & 95.63 \\
\hline Wqr & 33.18 & 32.60 & 34.61 & 37.80 & 36.21 & 39.27 & 39.15 & 38.86 & 39.12 \\
\hline Zoo & 48.01 & 85.67 & 72.15 & 49.23 & 72.15 & 48.08 & 87.48 & 62.64 & 23.67 \\
\hline Avg. & 66.81 & 75.63 & 74.21 & 69.03 & 74.73 & 71.33 & 76.76 & 73.80 & 68.41 \\
\hline
\end{tabular}


Table 4: Wilcoxon tests for comparison of ensemble learning and resampling techniques in the OVO scheme with CART as the base classifier. $R^{+}$corresponds to the sum of the ranks for ensemble learning and $R^{-}$for the resampling techniques.

\begin{tabular}{|c|c|c|c|c|c|c|c|}
\hline Comparison & $R^{+}$ & $R^{-}$ & $p$-value & Comparison & $R^{+}$ & $R^{-}$ & $p$-value \\
\hline UBA vs. RUS & 197.00 & 13.00 & 0.000629 & SBO vs. RUS & 157.00 & 53.00 & 0.052222 \\
\hline UBA vs. ROS & 135.50 & 74.50 & 0.295424 & SBO vs. ROS & 143.00 & 67.00 & 0.156004 \\
\hline UBA vs. SMOTE & 135.00 & 75.00 & 0.262722 & SBO vs. SMOTE & 179.00 & 31.00 & 0.005734 \\
\hline SBA vs. RUS & 144.00 & 66.00 & 0.145400 & SMB vs. RUS & 185.00 & 25.00 & 0.002821 \\
\hline SBA vs. ROS & 91.50 & 118.50 & 0.629162 & SMB vs. ROS & 131.00 & 79.00 & 0.331723 \\
\hline SBA vs. SMOTE & 158.00 & 52.00 & 0.022909 & SMB vs. SMOTE & 137.00 & 73.00 & 0.232226 \\
\hline RBO vs. RUS & 172.00 & 38.00 & 0.012374 & Easy vs. RUS & 167.00 & 43.00 & 0.020633 \\
\hline RBO vs. ROS & 127.00 & 83.00 & 0.411465 & Easy vs. ROS & 133.00 & 77.00 & 0.295878 \\
\hline RBO vs. SMOTE & 131.00 & 79.00 & 0.331723 & Easy vs. SMOTE & 133.00 & 77.00 & 0.295878 \\
\hline
\end{tabular}

with RUS and SMOTE, but achieves $p$-value slightly above the significance level for comparison with ROS. Similar situation can be observed for all of highlighted best-performing ensemble techniques. At the same time one must notice that the OVO-based ensemble learning does not damage the accuracies when compared to OVO single model learning with resampling. Therefore, we can summarize that ensemble learning with OVO decomposition is a preferred choice over OVO resampling techniques, never leading to reduced classification rates and often offering significant improvement for the multi-class imbalance classification problems with CART classifier.

\subsection{Evaluating Ensemble Approaches with BPNN as Base Classifier}

The complete results for the BPNN version are shown in Table 5.

In this case the trend is quite similar with the segment of CART, since most of the best results obtained in each data set are acquired by the ensemble learning approaches. Additionally, for the average results, SMB receives the best performance, followed closely by SBO. This is consistent with those obtained by CART. Therefore, we must highlight that the methodology of ensemble learning methods used to train the binary classifier in the OVO scheme achieve quite high quality of the average performance.

The Wilcoxon tests are carried out in Table 6, when BPNN is used as the base classifier.

In this case, all the null hypotheses of equivalence are rejected for the ensemble learning methods, since the largest $p$-value is equal to 0.043804 , 
Table 5: Average accuracy [\%] results for resampling and ensemble learning methods with BPNN as the base classifier. The best result is highlighted in bold.

\begin{tabular}{|c|c|c|c|c|c|c|c|c|c|}
\hline \multirow{2}{*}{ Data sets } & \multicolumn{3}{|c|}{ Resampling techniques } & \multicolumn{6}{|c|}{ Ensemble learning } \\
\hline & RUS & ROS & SMOTE & UBA & SBA & $\mathrm{RBO}$ & $\mathrm{SBO}$ & $\mathrm{SMB}$ & Easy \\
\hline Aut & 47.12 & 64.99 & 56.34 & 61.40 & 70.44 & 70.83 & 77.67 & 71.91 & 66.88 \\
\hline Bal & 70.81 & 89.11 & 85.93 & 88.18 & 91.07 & 85.80 & 94.12 & 86.08 & 88.28 \\
\hline Car & 61.78 & 72.10 & 66.98 & 77.17 & 80.41 & 90.27 & 97.98 & 95.93 & 91.79 \\
\hline Cle & 33.03 & 35.18 & 32.18 & 32.62 & 30.07 & 32.68 & 30.70 & 30.31 & 31.16 \\
\hline Con & 45.97 & 47.12 & 38.27 & 50.72 & 36.71 & 51.90 & 51.15 & 50.70 & 51.96 \\
\hline Der & 91.60 & 93.81 & 92.49 & 96.87 & 96.81 & 96.41 & 96.94 & 96.90 & 96.79 \\
\hline Fla & 56.96 & 60.07 & 54.11 & 65.24 & 59.61 & 63.38 & 62.28 & 63.75 & 65.05 \\
\hline Gla & 48.91 & 52.67 & 51.39 & 63.21 & 60.02 & 71.09 & 63.92 & 70.91 & 70.02 \\
\hline Hay & 60.17 & 61.84 & 53.36 & 71.06 & 66.53 & 77.78 & 82.49 & 78.38 & 78.71 \\
\hline Led & 68.48 & 67.28 & 67.25 & 73.64 & 73.04 & 71.54 & 72.15 & 70.81 & 72.02 \\
\hline Lym & 70.41 & 85.53 & 78.14 & 86.79 & 88.83 & 83.22 & 87.86 & 84.08 & 86.17 \\
\hline New & 90.04 & 94.89 & 92.80 & 97.70 & 96.52 & 97.07 & 96.22 & 97.19 & 97.93 \\
\hline Pag & 78.57 & 82.26 & 83.52 & 88.52 & 89.49 & 91.50 & 84.29 & 91.08 & 92.08 \\
\hline Sat & 86.24 & 86.58 & 85.47 & 87.38 & 86.99 & 89.30 & 88.30 & 89.71 & 89.55 \\
\hline Shu & 68.10 & 84.87 & 89.46 & 80.71 & 93.07 & 85.79 & 93.56 & 91.40 & 87.88 \\
\hline Spl & 85.76 & 86.70 & 83.67 & 90.34 & 90.32 & 89.73 & 91.32 & 90.55 & 89.78 \\
\hline Thy & 74.98 & 80.84 & 75.70 & 86.45 & 70.54 & 95.80 & 78.36 & 96.19 & 96.81 \\
\hline Win & 94.08 & 92.09 & 95.50 & 98.70 & 98.27 & 97.94 & 98.00 & 97.87 & 98.57 \\
\hline Wqr & 33.05 & 34.80 & 35.36 & 39.25 & 36.93 & 39.36 & 31.40 & 40.42 & 41.96 \\
\hline Zoo & 73.04 & 81.19 & 79.61 & 90.24 & 90.10 & 86.60 & 89.52 & 88.86 & 89.39 \\
\hline Avg. & 66.96 & 72.70 & 69.88 & 76.31 & 75.29 & 78.40 & 78.41 & 79.15 & 79.14 \\
\hline
\end{tabular}


Table 6: Wilcoxon tests for comparison of ensemble learning and resampling techniques in the OVO scheme with BPNN as the base classifier. $R^{+}$corresponds to the sum of the ranks for ensemble learning and $R^{-}$for the resampling techniques.

\begin{tabular}{|c|c|c|c|c|c|c|c|}
\hline Comparison & $R^{+}$ & $R^{-}$ & $p$-value & Comparison & $R^{+}$ & $R^{-}$ & $p$-value \\
\hline UBA vs. RUS & 209 & 1 & 0.000103 & SBO vs. RUS & 206 & 4 & 0.000163 \\
\hline UBA vs. ROS & 186 & 24 & 0.002495 & SBO vs. ROS & 186 & 24 & 0.002495 \\
\hline UBA vs. SMOTE & 197 & 13 & 0.000593 & SBO vs. SMOTE & 201 & 9 & 0.000338 \\
\hline SBA vs. RUS & 189 & 21 & 0.001713 & SMB vs. RUS & 208 & 2 & 0.00012 \\
\hline SBA vs. ROS & 159 & 51 & 0.043804 & SMB vs. ROS & 196 & 14 & 0.000681 \\
\hline SBA vs. SMOTE & 194 & 16 & 0.000892 & SMB vs. SMOTE & 208 & 2 & 0.00012 \\
\hline RBO vs. RUS & 209 & 1 & 0.000103 & Easy vs. RUS & 209 & 1 & 0.000103 \\
\hline RBO vs. ROS & 194 & 16 & 0.000892 & Easy vs. ROS & 199 & 11 & 0.000449 \\
\hline RBO vs. SMOTE & 205 & 5 & 0.000189 & Easy vs. SMOTE & 207 & 3 & 0.00014 \\
\hline
\end{tabular}

which is lower than our $\alpha$-value (0.05). This is much better than in the case of CART classifiers, as here we observe a globally statistically significant improvement regardless of the ensemble method used. This is a highly interesting observation, as commonly it was assumed that decision trees as weak learners should benefit the most from multiple classifier systems. However, one can see that combination of BPNNs works much better than combination of CARTs. Therefore, we can conclude that the ensemble learning approaches with OVO scheme for the multi-class imbalance classification problems outperform the OVO resampling techniques for BPNN classifier.

\subsection{Evaluating Ensemble Approaches with SVM as Base Classifier}

Finally, we show the complete results for the version of SVM in Table 7.

The average results obtained in this version are somewhat lower than in the case of BPNN, but it is consistent that the ensemble learning approaches improve the performance for the multi-class imbalance classification problems with the OVO scheme, comparing with the original resampling techniques. Additionally, once again SMB is considered as the best average performance, followed by Easy and RBO respectively.

Observing the statistical analysis shown in Table 8, the behavior of the ensemble learning techniques in the OVO scheme is generally better than those obtained by the resampling approaches.

According to the statistical results of Wilcoxon test, we can observe that RBO, SMB and Easy clearly outperform all of the conventional resampling 
Table 7: Average accuracy [\%] results for resampling and ensemble learning methods with SVM as the base classifier. The best result is highlighted in bold.

\begin{tabular}{|c|c|c|c|c|c|c|c|c|c|}
\hline \multirow{2}{*}{ Data sets } & \multicolumn{3}{|c|}{ Resampling techniques } & \multicolumn{6}{|c|}{ Ensemble learning } \\
\hline & RUS & ROS & SMOTE & UBA & SBA & $\mathrm{RBO}$ & $\mathrm{SBO}$ & SMB & Easy \\
\hline Aut & 47.86 & 56.74 & 58.01 & 52.30 & 57.63 & 60.71 & 67.34 & 67.51 & 60.41 \\
\hline Bal & 61.78 & 57.43 & 73.70 & 64.12 & 73.66 & 78.77 & 64.60 & 80.59 & 79.64 \\
\hline Car & 79.92 & 73.71 & 86.23 & 80.41 & 86.23 & 91.6 & 89.18 & 91.65 & 91.20 \\
\hline Cle & 30.78 & 24.20 & 33.23 & 33.46 & 33.99 & 32.07 & 31.61 & 32.13 & 34.53 \\
\hline Con & 50.37 & 46.32 & 48.40 & 50.76 & 48.40 & 50.12 & 41.61 & 50.35 & 50.85 \\
\hline Der & 97.46 & 95.49 & 96.21 & 97.66 & 96.21 & 97.49 & 96.53 & 97.72 & 97.34 \\
\hline Fla & 62.16 & 56.43 & 62.45 & 64.53 & 62.72 & 64.11 & 58.33 & 63.95 & 65.72 \\
\hline Gla & 47.83 & 53.14 & 50.81 & 49.53 & 51.86 & 68.67 & 60.81 & 68.37 & 66.18 \\
\hline Hay & 53.24 & 53.25 & 53.66 & 53.56 & 52.89 & 64.24 & 62.38 & 64.65 & 57.64 \\
\hline Led & 70.95 & 67.93 & 70.11 & 73.52 & 70.11 & 71.78 & 72.88 & 72.10 & 72.31 \\
\hline Lym & 83.12 & 89.26 & 85.16 & 85.94 & 88.50 & 83.35 & 87.80 & 82.96 & 87.35 \\
\hline New & 85.25 & 90.00 & 92.20 & 87.30 & 91.91 & 96.13 & 95.66 & 95.43 & 97.70 \\
\hline Pag & 71.30 & 76.84 & 79.63 & 73.15 & 80.46 & 89.87 & 83.84 & 90.95 & 90.74 \\
\hline Sat & 86.24 & 71.59 & 86.81 & 86.32 & 86.86 & 84.94 & 84.32 & 85.30 & 86.21 \\
\hline Shu & 68.27 & 94.15 & 94.73 & 74.64 & 94.70 & 77.97 & 99.33 & 88.72 & 85.46 \\
\hline Spl & 95.96 & 95.43 & 96.30 & 96.16 & 96.43 & 95.94 & 95.86 & 96.07 & 96.20 \\
\hline Thy & 68.27 & 79.49 & 82.61 & 70.48 & 82.74 & 91.11 & 78.74 & 92.46 & 93.67 \\
\hline Win & 96.37 & 96.18 & 96.57 & 97.07 & 96.57 & 97.25 & 98.05 & 97.88 & 97.62 \\
\hline Wqr & 37.20 & 35.53 & 38.64 & 38.57 & 38.83 & 40.63 & 24.67 & 39.41 & 38.92 \\
\hline Zoo & 87.16 & 88.47 & 90.29 & 88.77 & 90.87 & 90.14 & 91.68 & 88.38 & 90.07 \\
\hline Avg. & 69.07 & 70.08 & 73.79 & 70.91 & 74.08 & 76.34 & 74.26 & 77.33 & 76.99 \\
\hline
\end{tabular}


Table 8: Wilcoxon tests for comparison of ensemble learning and resampling techniques in the OVO scheme with SVM as the base classifier. $R^{+}$corresponds to the sum of the ranks for ensemble learning and $R^{-}$for the resampling techniques.

\begin{tabular}{llllllllll}
\hline Comparison & $R^{+}$ & $R^{-}$ & $p$-value & & Comparison & $R^{+}$ & $R^{-}$ & $p$-value \\
\cline { 1 - 5 } \cline { 6 - 8 } UBA vs. RUS & 210 & 0 & 0.000089 & & SBO vs. RUS & 166 & 44 & 0.022769 \\
UBA vs. ROS & 129 & 81 & 0.370261 & & SBO vs. ROS & 178 & 32 & 0.006425 \\
UBA vs. SMOTE & 62 & 148 & 0.108427 & & SBO vs. SMOTE & 122 & 88 & 0.525653 \\
SBA vs. RUS & 186 & 24 & 0.002495 & & SMB vs. RUS & 201 & 9 & 0.000338 \\
SBA vs. ROS & 196 & 14 & 0.000681 & & SMB vs. ROS & 191 & 19 & 0.001325 \\
SBA vs. SMOTE & 145.5 & 64.5 & 0.111769 & & SMB vs. SMOTE & 166 & 44 & 0.022769 \\
RBO vs. RUS & 197 & 13 & 0.000593 & & Easy vs. RUS & 207 & 3 & 0.00014 \\
RBO vs. ROS & 183 & 27 & 0.003592 & & Easy vs. ROS & 193 & 17 & 0.001019 \\
RBO vs. SMOTE & 165 & 45 & 0.025094 & & Easy vs. SMOTE & 186 & 24 & 0.002495 \\
\hline
\end{tabular}

Table 9: Friedman aligned-rank tests comparing the different ensemble learning methods with OVO scheme.

\begin{tabular}{llll}
\hline Ensemble learning & CART & BPNN & SVM \\
\hline UBA & $66.10(0.151106)$ & $71.10(0.168688)$ & $82.25(0.002173)$ \\
SBA & $75.15(0.018658)$ & $76.45(0.058981)$ & $73.60(0.025194)$ \\
RBO & $61.60(0.285839)$ & $58.35(1.000000)$ & $51.50(0.939695)$ \\
SBO & $61.25(0.285839)$ & $58.50(1.000000)$ & $67.35(0.091477)$ \\
SMB & 43.25 & $49.85(1.000000)$ & 43.55 \\
Easy & $55.65(0.285839)$ & 48.75 & $44.75(0.939695)$ \\
\hline
\end{tabular}

techniques. In addition, RUS is defeated by any ensemble learning approach. For ROS only UBA method does not deliver significant improvement. With regard to SMOTE the $p$-values observed for comparing with RBO, SMB and Easy are lower than our $\alpha$-value (0.05). Therefore, in the scenario of SVM we also can conclude that ensemble learning approaches outperform resampling techniques in the OVO scheme for the multi-class imbalance classification problems.

\subsection{Comparison of Different Used Ensemble Learning Techniques}

The goal of this study is to explore the validity of the ensemble learning approaches with OVO decomposition in the application of multi-class imbalance classification problems, since these techniques combine the single 
classifiers to be an ensemble, which is useful for addressing the binary-class imbalance problems. The facts found in this study support the goodness of the methodology of ensemble learning methods based on resampling techniques for improving the performance in dealing with the multi-class imbalance classification problems.

However, we also need to compare the different examined ensemble learning techniques among themselves in order to find the statistically best method for each base classifier. Using only averaged accuracy for such a selection can be misleading as we do not take into account the ranks among these methods for each dataset. Therefore, we need to conduct a statistical test over multiple datasets to choose the best combined classifier.

For this purpose we use the Friedman aligned-rank test in order to detect the highest-ranked methods. The results from this test are depicted in Table 9 .

When analyzing the aligned ranks for CART classifier we can clearly see that SMB method achieves the best results. Its rank is clearly lower than other methods. This is especially interesting when considering the fact of SBO scoring the best average accuracy results. But according to statistical analysis SMB method displays the most efficient and stable performance over all of datasets used.

For BPNN classifier we can see that the lowest ranks were obtained by Easy approach. However, differences between it and SMB are practically insignificant (48.75 vs. 49.85) thus allowing us to conclude that both of these methods display excellent performance for neural networks in mining multi-class imbalanced data.

Similar situation can be observed for SVM classifier. Here SMB method obtains the lowest ranks but differences between it and Easy method are negligible (43.55 vs. 44.75), showing that both of these methods are highly suitable for working with SVMs.

\subsection{Comparison with Ad-hoc Approaches for Multi-Class Imbalanced Data}

In order to put the obtained results into context we cannot refer only to OVO-based resampling techniques. There is a number of solutions already proposed in the literature for addressing skewed distributions in multiple classes. We decided to select two ensemble-based techniques from them as reference methods for our proposal. We have selected popular AdaBoost.NC [57], which is considered as one of the best approaches for handling multiclass imbalanced data. Additionally, we present results for recently intro- 
duced OVA-NBSVM [14] that combines binary modified SVMs using one-vsall technique.

For each base classifier examined we have selected a single best-performing ensemble technique according to the Friedman aligned-rank tests discussed in Section 5.4.This means that for comparison SMB for CART / SVM and Easy for BPNN are being used.

Accuracy results for examined benchmarks and methods are depicted in Table 10.

We will focus our discussion on comparison with reference methods, as accuracies of ensemble techniques for different base classifiers were discussed in previous sections.

OVA-NBSVM returned surprisingly the worst results from all of the examined methods. Only for a single dataset (Zoo) it was able to outperform all other approaches. This proves the lack of usefulness of OVA decomposition for multi-class problems with skewed distributions. OVA for some of class combinations additionally boosts the disproportion between classes thus making learning from small-sample classes very difficult. This can be observed for cases with high imbalance ratios and small classes like Pag and Wqr datasets. Here some of classes are very small when compared to others, which leads to a extremely high binary imbalance ratio when these classes are used as a positive and aggregation of remaining ones as a negative. For these cases OVA-NBSVM drops highly in comparison to all other discussed solutions.

AdaBoost.NC is a more demanding reference method, achieving best results on 4 datasets. However, on 16 remaining benchmarks one of the proposed ensemble techniques outperforms this boosting scheme. It allows us to conclude that empowering OVO decomposition with ensemble learning can be a highly efficient solution for the discussed problem, being able to deliver generally improved performance over most popular solutions from the literature.

To gain an additional insight into the performance of examined methods we have conducted a Wilcoxon test, outcomes of which are presented in Table 11.

Obtained $p$-values show that proposed ensemble techniques with BPNN and SVM are significantly better than OVA-NBSVM. For comparison with AdaBoost.NC only BPNN-based ensemble offer significantly superior results. When analyzing obtained $p$-values and accuracies for remaining methods we can see that they offer small but visible improvement over reference algo- 
Table 10: Complete average accuracy test results for the representative combinations (SMB-CART, Easy-BPNN and SMB-SVM) and the state-of-the-art approaches for mining multi-class imbalanced data (AdaBoost.NC and OVA-NBSVM). The best result is highlighted in bold.

\begin{tabular}{lccccc}
\hline Dataset & AdaBoost.NC & OVA-NBSVM & SMB-CART & Easy-BPNN & SMB-SVM \\
\hline Aut & $\mathbf{7 6 . 5 3}$ & 70.73 & 69.85 & 66.88 & 67.51 \\
Bal & 66.92 & 85.81 & 66.27 & $\mathbf{8 8 . 2 8}$ & 80.59 \\
Car & 85.08 & 54.83 & $\mathbf{9 7 . 5 6}$ & 91.79 & 91.65 \\
Cle & 30.33 & 31.11 & $\mathbf{3 3 . 1 8}$ & 31.16 & 32.13 \\
Con & 47.36 & 47.21 & 48.96 & $\mathbf{5 1 . 9 6}$ & 50.35 \\
Der & 95.00 & 96.45 & 94.65 & 96.79 & $\mathbf{9 7 . 7 2}$ \\
Fla & 60.57 & 54.45 & 62.60 & $\mathbf{6 5 . 0 5}$ & 63.95 \\
Gla & 69.89 & 61.96 & $\mathbf{7 2 . 5 9}$ & 70.02 & 68.37 \\
Hay & $\mathbf{8 5 . 3 9}$ & 76.42 & 85.24 & 78.71 & 64.65 \\
Led & $\mathbf{7 2 . 1 0}$ & 59.45 & 70.78 & 72.02 & $\mathbf{7 2 . 1 0}$ \\
Lym & 78.33 & 69.86 & 62.51 & $\mathbf{8 6 . 1 7}$ & 82.96 \\
New & 88.42 & 89.05 & 93.69 & $\mathbf{9 7 . 9 3}$ & 95.43 \\
Pag & 80.75 & 48.97 & $\mathbf{9 3 . 6 3}$ & 92.08 & 90.95 \\
Sat & 87.49 & 88.16 & $\mathbf{9 0 . 2 0}$ & 89.55 & 85.30 \\
Shu & $\mathbf{8 9 . 8 2}$ & 82.07 & 41.89 & 87.88 & 88.72 \\
Spl & 94.14 & 74.10 & 95.64 & 89.78 & $\mathbf{9 6 . 0 7}$ \\
Thy & 95.46 & 64.97 & $\mathbf{9 8 . 6 1}$ & 96.81 & 92.46 \\
Win & 93.13 & 97.58 & 96.60 & $\mathbf{9 8 . 5 7}$ & 97.88 \\
Wqr & 35.73 & 31.84 & 38.86 & $\mathbf{4 1 . 9 6}$ & 39.41 \\
Zoo & 86.67 & $\mathbf{9 0 . 6 2}$ & 62.64 & 89.39 & 88.38 \\
\hline Avg. & 75.96 & 68.78 & 73.80 & $\mathbf{7 9 . 1 4}$ & 77.33 \\
\hline
\end{tabular}


Table 11: Wilcoxon tests for comparison of the representative ensemble learning with OVO scheme and the state-of-the-art methods for multi-class imbalanced data. $R^{+}$corresponds to the sum of the ranks for the representative ensemble learning and $R^{-}$for the state-ofthe-art methods.

\begin{tabular}{lccl}
\hline Comparison & $R^{+}$ & $R^{-}$ & $p$-value \\
\hline SMB-CART vs. AdaBoost.NC & 128 & 82 & 0.390533 \\
Easy-BPNN vs. AdaBoost.NC & 162 & 48 & 0.033340 \\
SMB-SVM vs. AdaBoost.NC & 150 & 60 & 0.092963 \\
SMB-CART vs. OVA-NBSVM & 146 & 64 & 0.125859 \\
Easy-BPNN vs. OVA-NBSVM & 198 & 12 & 0.000517 \\
SMB-SVM vs. OVA-NBSVM & 172 & 38 & 0.012374 \\
\hline
\end{tabular}

rithms. In conclusion it must be noted that the proposed combination of OVO and pairwise ensemble learning can outperform state-of-the-art methods for multi-class imbalance on a variety of datasets, thus being a worthwhile choice for such problems.

\section{Lessons Learned}

In order to summarize this manuscript let us present three main points capturing the most important research findings.

1. The role of ensemble learning method. Obtained results allow us to observe the existence of a trend among six examined ensemble learning techniques. Regardless of the used base classifier SMB and Easy methods delivered the best performance both in terms of averaged accuracy and ranking statistical tests. This makes them the most universal ensemble techniques that should always be examined in combination with OVO decomposition when imbalanced multi-class problems are being faced. Additionally, RBO and SBO techniques tend to deliver good results for some of the datasets, thus making them a reasonable second choice if time allows for a more compound experimental study.

2. The role of base classifier. The choice of a base classifier had a significant impact on the observed classification accuracies. Surprisingly, used decision tree model (CART) returned least satisfactory improvement when used in ensemble setting. This is contrary to numerous statements in the literature, reporting excellent properties of tree models as weak classifiers for combination. This may be explained by high 
sensitivity of CART model to skewed distributions. SVM worked very well with most of the ensemble techniques, achieving improved classification rates in most of scenarios. BPNN was found to be the best working model in ensemble setting, achieving statistically superior results for any kind of used committee approach.

3. Comparison with resampling and ad-hoc solutions. The proposed empowering of OVO decomposition with pairwise ensemble learning achieved highly competitive results when compared with standard OVO with resampling techniques applied. Using compound learners for each pair of classes can lead to a better capturing of their local specifics, higher robustness to imbalance and improved final multi-class recognition rates. Additionally, proposed ensemble-based OVO is able to outperform state-of-the-art methods for multi-class imbalanced data, especially those using OVA solutions.

\section{Conclusions and Future Works}

In this paper we have proposed to improve the performance of binary decomposition used for multi-class imbalanced problems by applying ensemble learning paradigm to each sub-problem. This way we are able to exploit highly efficient combined classification approaches that were so far restricted to binary scenarios. Their proven efficacy in two-class imbalanced tasks motivated us to extend their area of applicability to much more challenging scenarios, where multiple majority and minority classes are present and the relationships between them are no longer obvious. As we wanted to capture pairwise relations between objects we focused one OVO decomposition, as it does not affect the distributions of positive and negative classes.

To check the flexibility of the proposed approach we have tested it with three different base classifiers: CART, BPNN and SVM. Experimental study, backed-up by a thorough statistical analysis indicate that it is possible to significantly boost the OVO approach performance for multi-class imbalanced data when enhancing it with ensemble classifiers. Regardless of the used committee approach in most cases we were able to outperform traditional OVO approach utilizing single classifiers with pre-processing algorithms, as well as state-of-the-art multi-class solutions for skewed data.

Obtained results allow us to formulate recommendations for selecting ensemble schemes. SMB technique is the best choice when considering CART and SVM as base classifiers. At the same time BPNN should be used with 
Easy approach. As result differences between several methods are not high, we point out the high effectiveness of Easy, SMB and RBO ensemble methods for empowering OVO techniques in multi-class imbalance scenarios.

We highlighted the effectiveness of synergy between decomposition strategy and ensemble learning in the multi-class imbalanced datasets. Above all, we must emphasize that our work provides the basis for addressing the multiclass imbalance classification problems with a combination of decomposition and multiple classifier approaches.

In our study we only presented the VOTE aggregation strategy. Therefore, it is interesting to develop the analysis of the other aggregations in the OVO scheme and trained combiners like Error-Correcting Output Codes or Decision Templates. Additionally, we would like to extend our proposal to include recent findings in OVO decomposition that take into consideration the dynamic classifier selection [24] and competence-based weighting [26] to remove the non-competent classifiers from the pool.

\section{Acknowledgments}

Zhongliang Zhang was supported by the National Science Foundation of China (NSFC Proj. 61273204) and CSC Scholarship Program (CSC NO. 201406080059).

Bartosz Krawczyk was supported by the Polish National Science Center under the grant no. DEC-2013/09/B/ST6/02264.

Salvador Garcia and Francisco Herrera were partially supported by the Spanish Ministry of Education and Science under Project TIN2014-57251-P and the Andalusian Research Plan P10-TIC-6858, P11-TIC-7765.

Alejandro Rosales-Pérez was supported by the CONACyT grant 329013.

\section{References}

[1] M.H.B. Abidine and B. Fergani. A new multi-class wsvm classification to imbalanced human activity dataset. Journal of computers, 9(7):15601565, 2014.

[2] R. Baeza-Yates and B. Ribeiro-Neto. Modern information retrieval. ACM press, New York, 1999.

[3] R. Barandela, R.M. Valdovinos, and J.S. Sanchez. New applications of ensembles of classifiers. Pattern Analysis \& Applications, 6(3):245-256, 2003. 
[4] G.E.A.P.A. Batista, R.C. Prati, and M.C. Monard. A study of the behavior of several methods for balancing machine learning training data. ACM SIGKDD Explorations Newsletter, 6(1):20-29, 2004.

[5] J. Blaszczynski and J. Stefanowski. Neighbourhood sampling in bagging for imbalanced data. Neurocomputing, 150:529-542, 2015.

[6] L. Breiman, J. Friedman, C.J. Stone, and R.A. Olshen. Classification and regression trees. CRC press, 1984.

[7] L. Cerf, D. Gay, N. Selmaoui-Folcher, B. Cremilleux, and J.F. Boulicaut. Parameter-free classification in multi-class imbalanced data sets. Data 83 Knowledge Engineering, 87:109-129, 2013.

[8] N.V. Chawla, K.W. Bowyer, L.O. Hall, and W.P. Kegelmeyer. Smote: synthetic minority over-sampling technique. Journal of artificial intelligence research, 16:321-357, 2002.

[9] N.V. Chawla, N. Japkowicz, and A. Kotcz. Editorial: special issue on learning from imbalanced data sets. ACM SIGKDD Explorations Newsletter, 6(1):1-6, 2004.

[10] N.V. Chawla, A. Lazarevic, L.O. Hall, and K.W. Bowyer. Knowledge Discovery in Databases: PKDD 2003, volume 2838 of Lecture Notes in Computer Science, chapter SMOTEBoost: Improving prediction of the minority class in boosting, pages 107-119. Springer-Verlag, 2003.

[11] D.A. Cieslak, T.R. Hoens, N.V. Chawla, and W.P. Kegelmeyer. Hellinger distance decision trees are robust and skew-insensitive. Data Mining and Knowledge Discovery, 24(1):136-158, 2012.

[12] W.M. Czarnecki and J. Tabor. Two ellipsoid support vector machines. Expert Syst. Appl., 41(18):8211-8224, 2014.

[13] W.M. Czarnecki and J. Tabor. Multithreshold entropy linear classifier: Theory and applications. Expert Syst. Appl., 42(13):5591-5606, 2015.

[14] S. Datta and S. Das. Near-bayesian support vector machines for imbalanced data classification with equal or unequal misclassification costs. Neural Networks, 70:39-52, 2015. 
[15] J.-F. Díez-Pastor, J.-J. Rodríguez Diez, C. García-Osorio, and L. I. Kuncheva. Random balance: Ensembles of variable priors classifiers for imbalanced data. Knowl.-Based Syst., 85:96-111, 2015.

[16] T. Fawcett. An introduction to roc analysis. Pattern recognition letters, 27(8):861-874, 2006.

[17] R. Feraud and F. Clerot. A methodology to explain neural network classification. Neural Networks, 15(2):237-246, 2002.

[18] A. Fernandez, V. Lopez, M. Galar, M.J. del Jesus, and F. Herrera. Analysing the classification of imbalanced data-sets with multiple classes: Binarization techniques and ad-hoc approaches. KnowledgeBased Systems, 42:97-110, 2013.

[19] F. Fernandez-Navarro, C. Hervas-Martnez, and P.A. Gutierrez. A dynamic over-sampling procedure based on sensitivity for multi-class problems. Pattern Recognition, 44(8):1821-1833, 2011.

[20] C. Ferri, J. Hernandez-Orallo, and R. Modroiu. An experimental comparison of performance measures for classification. Pattern Recognition Letters, 30(1):27-38, 2009.

[21] J.H. Friedman. Another approach to polychotomous classification. Technical report: http://www. stat. stanford. edu/jhf, 1996.

[22] M. Galar, A. Fernndez, E. Barrenechea, H. Bustince, and F. Herrera. An overview of ensemble methods for binary classifiers in multi-class problems: Experimental study on one-vs-one and one-vs-all schemes. Pattern Recognition, 44(8):1761-1776, 2011.

[23] M. Galar, A. Fernandez, E. Barrenechea, H. Bustince, and F. Herrera. A review on ensembles for the class imbalance problem: bagging, boosting-, and hybrid-based approaches. IEEE Transactions on Systems, Man, and Cybernetics-Part C: Applications and Reviews, 42(4):463-484, 2012.

[24] M. Galar, A. Fernndez, E. Barrenechea, H. Bustince, and F. Herrera. Dynamic classifier selection for one-vs-one strategy: Avoiding non-competent classifiers. Pattern Recognition, 46(12):3412-3424, 2013. 
[25] M. Galar, A. Fernandez, E. Barrenechea, and F. Herrera. Empowering difficult classes with a similarity-based aggregation in multi-class classification problems. Information Sciences, 264:135-157, 2014.

[26] M. Galar, A. Fernndez, E. Barrenechea, and F. Herrera. Drcw-ovo: Distance-based relative competence weighting combination for one-vsone strategy in multi-class problems. Pattern Recognition, 48(1):28-42, 2015 .

[27] S. Garcia, J. Derrac, I. Triguero, C.J. Carmona, and F. Herrera. Evolutionary-based selection of generalized instances for imbalanced classification. Knowledge-Based Systems, 25(1):3-12, 2012.

[28] S. Garcia, A. Fernandez, J. Luengo, and F. Herrera. Advanced nonparametric tests for multiple comparisons in the design of experiments in computational intelligence and data mining: Experimental analysis of power. Information Sciences, 180(10):2044-2064, 2010.

[29] S. García, J. Luengo, and F. Herrera. Tutorial on practical tips of the most influential data preprocessing algorithms in data mining. Knowledge-Based Systems, 98:1 - 29, 2016.

[30] H. Guo, Y. Li, Y. Li, X. Liu, and J. Li. Bpso-adaboost-knn ensemble learning algorithm for multi-class imbalanced data classification. Eng. Appl. of AI, 49:176-193, 2016.

[31] H. Haibo and E.A. Garcia. Learning from imbalanced data. IEEE Transactions on Knowledge and Data Engineering, 21(9):1263-1284, 2009.

[32] D.J. Hand and R.J. Till. A simple generalisation of the area under the ROC curve for multiple class classification problems. Machine Learning, 45(2):171-186, 2001.

[33] S. Kang, S. Cho, and P. Kang. Constructing a multi-class classifier using one-against-one approach with different binary classifiers. Neurocomputing, 149:677-682, 2015.

[34] B. Krawczyk. Combining one-vs-one decomposition and ensemble learning for multi-class imbalanced data. In R. Burduk, K. Jackowski, M. Kurzyński, M. Woźniak, and A. Żołnierek, editors, Proceedings of the 
9th International Conference on Computer Recognition Systems CORES 2015, pages 27-36, 2016.

[35] B. Krawczyk, M. Galar, Ł Jeleń, and F. Herrera. Evolutionary undersampling boosting for imbalanced classification of breast cancer malignancy. Applied Soft Computing, 2015.

[36] B. Krawczyk, M. Woźniak, and G. Schaefer. Cost-sensitive decision tree ensembles for effective imbalanced classification. Appl. Soft Comput., 14:554-562, 2014.

[37] M. Kurzyński and M. Woźniak. Combining classifiers under probabilistic models: experimental comparative analysis of methods. Expert Systems, 29(4):374-393, 2012.

[38] Y. Li, H. Guo, X. Liu, Y. Li, and J. Li. Adapted ensemble classification algorithm based on multiple classifier system and feature selection for classifying multi-class imbalanced data. Knowl.-Based Syst., 94:88-104, 2016.

[39] B. Liu, Z.F. Hao, and E.C.C. Tsang. Nesting one-against-one algorithm based on svms for pattern classification. IEEE Transactions on Neural Networks, 19(12):2044-2052, 2008.

[40] X.Y. Liu, J.X. Wu, and Z.H. Zhou. Exploratory undersampling for class-imbalance learning. IEEE Transactions on Systems, Man, and Cybernetics-Part B: Cybernetics, 39(2):539-550, 2009.

[41] V. Lopez, A. Fernandez, S. Garcia, V. Palade, and F. Herrera. An insight into classification with imbalanced data: Empirical results and current trends on using data intrinsic characteristics. Information Sciences, 250:113-141, 2013.

[42] V. Lopez, A. Fernandez, and F. Herrera. On the importance of the validation technique for classification with imbalanced datasets: Addressing covariate shift when data is skewed. Information Sciences, 257:1-13, 2014 .

[43] J.G. Moreno-Torres, J.A. Saez, and F. Herrera. Study on the impact of partition-induced dataset shift on k-fold cross-validation. IEEE Transactions on Neural Networks and Learning Systems, 23(8):1304-1312, 2012. 
[44] W. Penar and M. Woźniak. Cost-sensitive methods of constructing hierarchical classifiers. Expert Systems, 27(3):146-155, 2010.

[45] M. D. Pérez-Godoy, A. J. Rivera, C. J. Carmona, and del Jesús.

[46] W. Prachuabsupakij and N. Soonthornphisaj. Clustering and combined sampling approaches for multi-class imbalanced data classification. $A d$ vances in Information Technology and Industry Applications, 136:717$724,2012$.

[47] P. Pramokchon and P. Piamsanga. Recent Advances in Information and Communication Technology, volume 265 of Advances in Intelligent Systems and Computing, chapter Reducing Effects of Class Imbalance Distribution in Multi-class Text Categorization, pages 263-272. SpringerVerlag, 2014.

[48] J. A. Sáez, B. Krawczyk, and M. Woźniak. Analyzing the oversampling of different classes and types of examples in multi-class imbalanced datasets. Pattern Recognion. 10.1016/j.patcog.2016.03.012.

[49] C. Seiffert, T.M. Khoshgoftaar, J. Van Hulse, and A. Napolitano. Rusboost: A hybrid approach to alleviating class imbalance. IEEE Transactions on Systems, Man and Cybernetics-Part A: Systems and Humans, 40(1):185-197, 2010.

[50] Y. Sun, A.K.C. Wong, and M.S. Kamel. Classification of imbalanced data: A review. Journal of Pattern Recognition and Artificial Intelligence, 23(4):687-719, 2009.

[51] M.A. Tahir, J. Kittler, and F. Yan. Inverse random under sampling for class imbalance problem and its application to multi-label classification. Pattern Recognition, 45(10):3738-3750, 2012.

[52] Y.C. Tang, Y.Q. Zhang, N.V. Chawla, and S. Krasser. Svms modeling for highly imbalanced classification. IEEE Transactions on Systems, Man, and Cybernetics-Part B: Cybernetics, 39(1):281-288, 2009.

[53] P. Trajdos and M. Kurzyński. An extension of multi-label binary relevance models based on randomized reference classifier and local fuzzy 
confusion matrix. In Intelligent Data Engineering and Automated Learning - IDEAL 2015 - 16th International Conference Wroclaw, Poland, October 14-16, 2015, Proceedings, pages 69-76, 2015.

[54] V. Vapnik. Statistical learning theory. Wiley, New York, 1998.

[55] P. Vorraboot, S. Rasmequan, K. Chinnasarn, and C. Lursinsap. Improving classification rate constrained to imbalanced data between overlapped and non-overlapped regions by hybrid algorithms. Neurocomputing, 152:429-443, 2015.

[56] S. Wang and X. Yao. Diversity analysis on imbalanced data sets by using ensemble models. In IEEE Symposium on Computational Intelligence and Data Mining, 2009, pages 324-331, 2009.

[57] S. Wang and X. Yao. Multiclass imbalance problems: Analysis and potential solutions. IEEE Transactions on Systems, Man, and CyberneticsPart B: Cybernetics, 42(4):1119-1130, 2012.

[58] G. Weiss and Y. Tian. Maximizing classifier utility when there are data acquisition and modeling costs. Data Mining and Knowledge Discovery, $17(2): 253-282,2008$.

[59] G.M. Weiss. Mining with rarity: a unifying framework. ACM SIGKDD Explorations Newsletter, 6(1):7-19, 2004.

[60] F. Wilcoxon. Individual comparisons by ranking methods. Biometrics bulletin, 1(6):80-83, 1945.

[61] T. Wilk and M. Woźniak. Soft computing methods applied to combination of one-class classifiers. Neurocomputing, 75(1):185-193, 2012.

[62] M. Woźniak, M. Graña, and E. Corchado. A survey of multiple classifier systems as hybrid systems. Information Fusion, 16:3-17, 2014.

[63] Z.H. Zhou and X.Y. Liu. On multi-class cost-sensitive learning. Computational Intelligence, 26(3):232257, 2010. 